\title{
Modeling of Grazing-Incidence X-ray Diffraction from Naphthyl End-Capped Oligothiophenes in Organic Field-Effect Transistors
}

Winokur, Michael J.; Huss-Hansen, Mathias K.; Lauritzen, Andreas E.; Torkkeli, Mika; Kjelstrup-Hansen, Jakob; Knaapila, Matti

\section{Published in:}

Crystal Growth and Design

Link to article, DOI:

10.1021/acs.cgd.0c00281

Publication date:

2020

Document Version

Peer reviewed version

Link back to DTU Orbit

Citation (APA):

Winokur, M. J., Huss-Hansen, M. K., Lauritzen, A. E., Torkkeli, M., Kjelstrup-Hansen, J., \& Knaapila, M. (2020). Modeling of Grazing-Incidence X-ray Diffraction from Naphthyl End-Capped Oligothiophenes in Organic FieldEffect Transistors. Crystal Growth and Design, 20(6), 3968-3978. https://doi.org/10.1021/acs.cgd.0c00281

\section{General rights}

Copyright and moral rights for the publications made accessible in the public portal are retained by the authors and/or other copyright owners and it is a condition of accessing publications that users recognise and abide by the legal requirements associated with these rights.

- Users may download and print one copy of any publication from the public portal for the purpose of private study or research.

- You may not further distribute the material or use it for any profit-making activity or commercial gain

- You may freely distribute the URL identifying the publication in the public portal 


\title{
Modelling of Grazing-Incidence X-ray Diffraction from Naphthyl End-capped Oligothiophenes in Organic Field-Effect Transistors
}

\author{
Michael J Winokur, ${ }^{* \dagger, \ddagger}$ Mathias K. Huss-Hansen, ${ }^{\ddagger}$ Andreas E. Lauritzen, ${ }^{9, \ddagger}$ \\ Mika Torkkeli, ${ }^{\ddagger}$ Jakob Kjelstrup-Hansen, ${ }^{\S}$ and Matti Knaapila ${ }^{\ddagger}$ \\ $\dagger$ Department of Physics, University of Wisconsin, Madison, Wisconsin 53706 \\ $\ddagger$ Department of Physics, Technical University of Denmark, 2800 Kgs. Lyngby, Denmark \\ \Department of Physics, University of Oxford, OX1 3PU Oxford, United Kingdom \\ $\S N a n o S Y D$, Mads Clausen Institute, University of Southern Denmark, 6400 Sønderborg, \\ Denmark
}

E-mail: mwinokur@wisc.edu

Phone: +1 (608)263 7475

\begin{abstract}
The structure of two naphthylene-capped oligothiophene, 5, 5'-bis(naphth-2-yl)-2,2'bi- and tri- thiophene, thin-film field-effect transistor assemblies has been studied using modeling in conjunction with grazing incidence x-ray diffraction. Although the well known herringbone molecular packing motif is observed in these films for both compounds, density functional calculations and molecular mechanics modeling give evidence for a local polymorphic ordering in which these molecules can be flipped $180^{\circ}$ about the long axis. In one case, that of the oligothiophene trimer, a disordered surface induced phase is observed. Prospective structural models are tested and refined using


various supercell constructions optimized by molecular mechanics prior to structure refinements of the thin-film scattering data.

\section{Introduction}

Deciphering the structure within organic thin films remains an important goal for fully understanding the behavior of organic electronic (OE) devices ${ }^{1}$ including organic field-effect transistors $^{2}$ (OFETs), organic photovoltaics (OPVs) and diverse OFET based sensors. ${ }^{3}$ Knowledge of the bulk structure often provides a useful reference point but the actual structural ordering in thin-film OE devices can differ in very important ways. ${ }^{4}$ This result should not be surprising given that interfacial interactions are more important in thin films. Ever present in OEs is the inherent anisotropy of the molecular level forces combined with non-equilibrium growth conditions. ${ }^{5}$ This gives rise to a variety of effects including so called surface induced phases (SIPs) and polymorphism. ${ }^{6,7}$ Working devices may even undergo evolution in the structural order during device operation. ${ }^{8}$

From a structural standpoint the family of dinaphthylene terminated oligothiphenes (NaTx's, with $\mathrm{x}=2$ and 3 shown in Fig. 1 respectively) represents a limiting case of a very stiff rod-like moiety with respect to the intramolecular structure in combination with much weaker Van der Waal (VdW) intermolecular interactions. Both the thiophene dimer (NaT2) and trimer (NaT3) compounds form single crystals in bulk suitable for crystal refinement. ${ }^{9}$ NaT2 yields a classic structure ${ }^{10}$ in which the long axes of near neighbors are aligned parallel to yield a two-dimensional layer structure along the $b$ - $c$ plane as depicted in the top panel of Fig. 2. Locally, within each layer, two molecules pack into a repeating herringbone motif. NaT2 is found to be monoclinic with P2 $2_{1}$ symmetry. NaT3 is more complicated ${ }^{11}$ and this single-crystal refinement identifies an unusually large three layer repeat in which the middle layer adopts a slightly different tilt angle of the NaT3 long axis in comparison to that in the two adjoining layers. This outcome may be due the distinct differences in 
the intrinsic molecular anisotropy between NaT2 and NaT3. As Fig. 1a shows NaT2, with an even number of thiophene rings, has a fundamentally different molecular symmetry from those NaTx compounds having an odd number of rings (such as NaT3 shown in Fig. 1b). Planar NaT2 has inversion symmetry with respect to the black dot signifying the geometric center while NaT3 has mirror symmetry about the axis which bisects the central thiophene ring. The red arrows are intended to further visualize this distinction with regards to the core thiophene units.

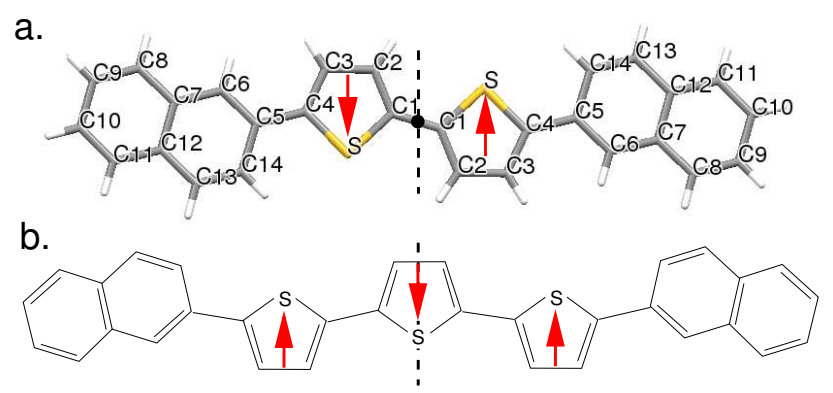

Figure 1: Sketches of the molecular structures for NaT2 (top) and NaT3 (bottom) according to single crystal refinements. ${ }^{9}$ The red arrows specify the orientation of the thiophene rings and, in combination with the vertical dashed line, show the different symmetry of NaTx molecules having an even or odd number of thiophene rings.

Thin films, the typical configuration for both OFETs and OPVs, are more difficult to assess by direct methods. There are recent reports ${ }^{12}$ highlighting the use molecular modeling in combination with refinement of novel structures in polymer OE thin films. Notable in Ref. 12 is the observation of an SIP having triclinic symmetry and a unit cell containing just a single molecule. To validate this model the authors employed density functional theory (DFT) calculations in tandem with a more traditional structure refinement (SR). In their SR the individual lattice reflections and their intensities were initially tabulated and then refined using a conventional crystallographic $R$-factor with appropriate constraints in the molecular geometry. This method is certainly sound but has limited use in cases where the crystallite sizes become small, the unit cell lattice parameters are large and/or there is appreciable structural disorder. At issue is the appearance of overlapping peaks and broad scattering features. In principal prospective structures and refinements could employ the 
full 2D grazing-incidence x-ray diffraction (GIXRD) intensity maps but this approach can be computationally cumbersome and requires considerable effort to effectively treat background contributions and other experimental scattering artifacts.

In this paper we report detail structural analyses of NaT2 and NaT3 thin-films in OFETs using both molecular modeling and a customized refinement of the GIXRD data with 1D "layer-line" scans of the 2D detector data. This work extends prior characterization and device studies of NaT2 ${ }^{13,14}$ and NaT3. ${ }^{15} \mathrm{NaT} 2$ thin films adopt a structure similar to that of the reported bulk phase whereas NaT3 thin films yield a much more disordered layer structure which we identify here as a substrate SIP. Molecular modeling calculations indicate that local intermolecular packing disorder is an important complicating factor in this class of compounds. Model DFT calculations are used to validate two distinct molecular packing motifs that are very close in their total energy. The only difference is that, with respect to near-neighbor pairs forming the classic herringbone motif, one molecule is flipped $180^{\circ}$ about its long axis.

To accommodate this proposed alternative packing into subsequent structural refinements we employ a preliminary step whereby various supercell (SC) model structures are first optimized via empirical force-fields in a zero temperature molecular mechanics (MM) calculation. These starting structures are then used in the subsequent refinements of the GIXRD 2D data. GIRXD 2D maps are computationally simulated and then compared directly to the intensities along individual 1D $k \ell$ "layer-lines" (because of cylindrical powder averaging these line are really cylinders which appear as lines because of the projection of the Ewald sphere onto the area detector). The structure factor (SF) refinements yield reasonably good agreement with the experimental data. A major observation is that introduction of local disorder in NaT2 can actually improve the agreement slightly and this structure is sound from an energetic standpoint as well. Likely consequences of this disorder within the unit cell with regards to OFET transport properties are discussed. For NaT3 we are able to substantiate a qualitative thin-film model with a molecular packing closer to NaT2 rather 
than that of NaT3 crystals.

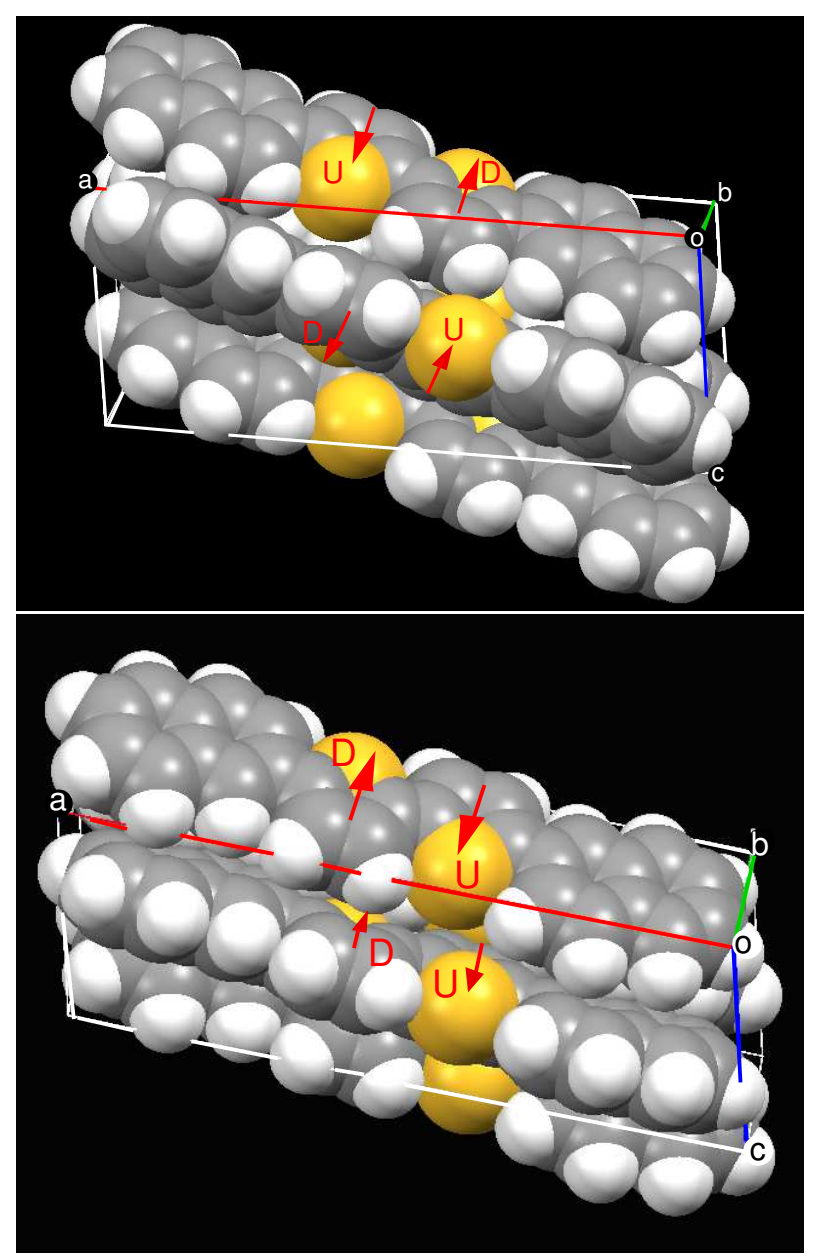

Figure 2: Top: The unit cell packing of bulk NaT2 (Model A). Bottom: A proposed NaT2 (or NaT3) packing polymorph (Model B) which, according to molecular modeling and density functional theory calculations, is nearly degenerate in energy. The red arrows and letter designation (i.e., $\mathrm{U}$ and $\mathrm{D}$ ) show the relationships between these two structure. All that is needed is to flip one molecule $180^{\circ}$ among the pair about its long axis.

\section{Results and Discusision}

\section{Experimental Grazing Incidence XRD}

Experimental room temperature GIXRD 2D maps for NaT2 and NaT3 are shown at top in Figs. 3 and 4 respectively. These figures are composite images from two separate 2D maps recorded at differing detector angles as noted previously. We first discuss the NaT2 
results. The NaT2 thin films exhibit a textured crystalline structure with peak positions and intensities comparable to those reported for the bulk crystal. All films exhibit a strong, uniaxial alignment so that the reciprocal $\mathrm{a}^{*}$-axis is parallel to the $q_{z}$ axis (i.e., perpendicular to the substrate surface) and perpendicular to the $b-c$ plane of the conventional molecular unit cells. There is an isotropic ordering of these crystallites about this a* axis. With this texture all $(h k \ell)$ peaks with nonzero $h$ or $k$ actually form concentric rings of scattering about the $q_{z}$ axis. A very modest arcing of the reflections is also seen and results from crystalline regions not perfectly parallel to the film surface.

The observed Bragg peaks are noticeably anisotropic in their relative peak widths along various scattering directions. Parallel to the film surface they are much sharper than in the perpendicular direction. The corresponding crystal size anisotropy is even more pronounced because of the significant contributions by the beam and film widths in the $q_{x y}$ direction. ${ }^{14}$ For NaT2, using a simple application of the Scherrer formula $\xi=5.66 / \Delta q$ where $\Delta q$ is the full width at half maximum of a scattering peak, yields nominal crystallite coherence lengths $\xi$ in excess of $200 \AA$ for the $b$-c plane (in-plane) and just $70 \AA$ along the $\mathrm{a}^{*}$ direction (out-of-plane). The in-plane NaT3 coherence length is similar to that of NaT2. Detailed assessments of crystal sizes for NaT2 and NaT3 samples are reported in Table 3 in Ref. 14 and in Table 1 of Ref. 15 respectively. These crystal sizes are generally insensitive to film thickness. The overall extent of crystallinity is high, perhaps over $90 \%$, but without a quantitative analysis this is only an estimate.

For NaT2, given the overall molecular length, this implies that a nominal crystal domain of just four or so repeats along the $a$-axis and, notably, this is on the order of the film surface roughness. With such a small number of layers there will be considerable lattice fringing and so underlies the relatively large wings of scattering as seen along the $q_{z}$ direction.

These well-defined, distinct Bragg peaks are superimposed on a characteristic background which includes weaker diffuse scattering from the thermal motion and static disorder of the oligothiophenes. Finally there are very weak scattering intensities suggestive of a second 
Figure 3: Top: A typical room temperature NaT2 GIXRD 2D composite image is shown in which images at two detector positions have been merged (at $q_{x y}=1.40 \AA^{-1}$ ). The various "layer-lines" are labeled with their respective Miller indices. Bottom: Simulated NaT2 2D GIXRD image from refinement of the $8 \times 1 \times 1$ NaT2 "no flip" model (see text) at room temperature. The area to the left of the yellow dashed lines is typically not observable in the experimental 2D maps. In both cases the image color scales are based on the square root of the scattering intensities.
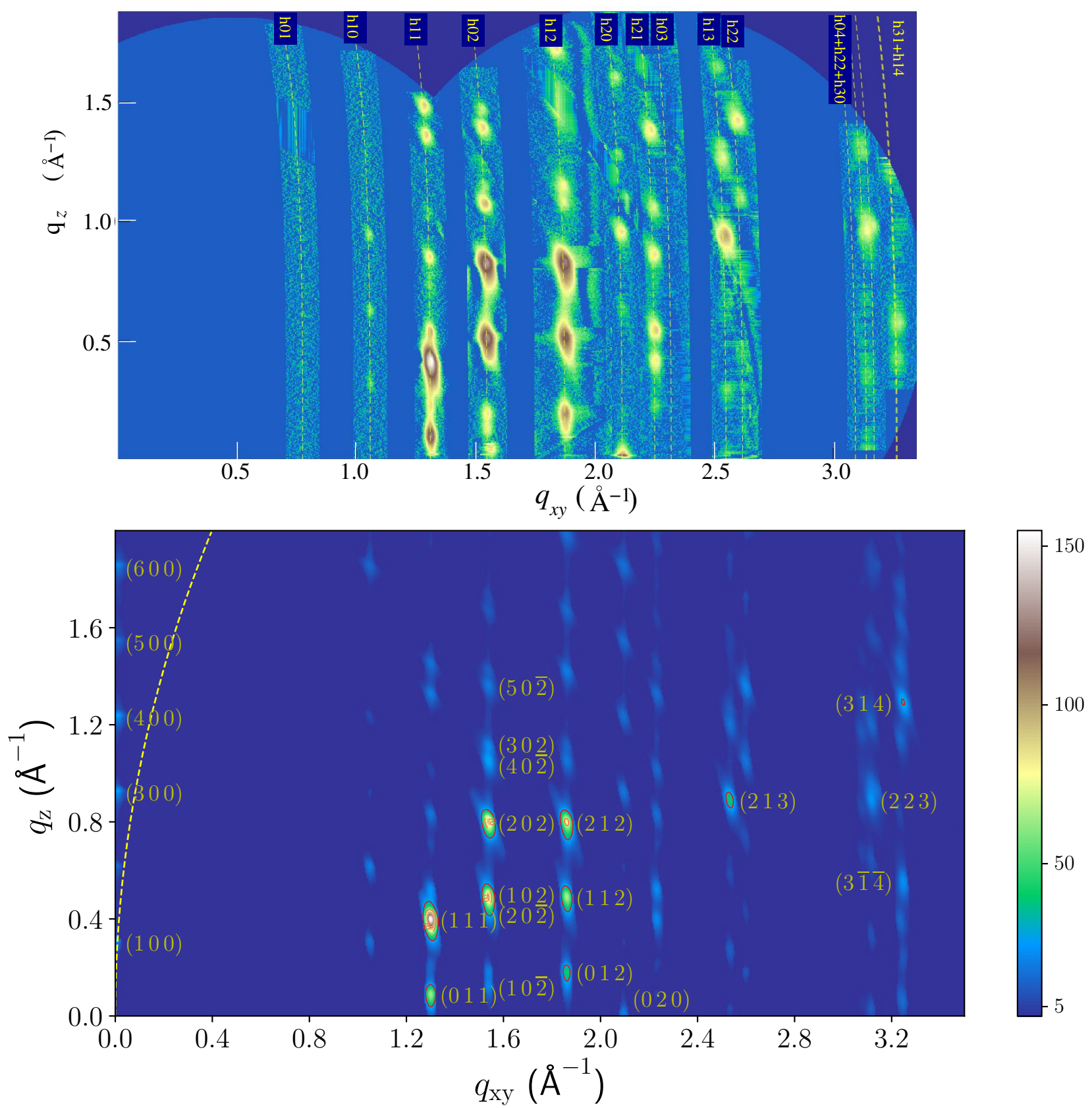
Figure 4: Top: A typical room temperature NaT3 GIXRD 2D composite image using the procedure as stated in Fig. 3. Bottom: Simulated NaT3 2D GIXRD image from refinement of a $6 \times 2 \times 2$ supercell (with $\beta=101.0^{\circ}$ ) containing a large amount of disorder in the form of $180^{\circ}$ flips about the long axis of the molecule. The area to the left of the yellow dashed lines is typically not observable in the experimental 2D maps. In both cases the image color scales are based on the square root of the scattering intensities. 
orientation of the NaT2.

Simulated 2D images and the explicit GIXRD data comparisons from this sample at elevated temperatures, at $343 \mathrm{~K}\left(70{ }^{\circ} \mathrm{C}\right)$ and at $393 \mathrm{~K}\left(120^{\circ} \mathrm{C}\right)$, are included in the supporting materials (i.e., series C, D shown in S10-S15 and G shown in S18-S23). The only noticeable changes are the monotonic shifts in the lattice constants consistent with a nominal thermal expansion within this $\mathrm{VdW}$ bonded solid. Thus, with respect to room temperature, most peaks shift to lower wavevector but, because there are also changes in the unit cell angle $\beta$, some peaks actually shift to higher wavevector. The full set of unit cell parameter results appears in Table 1

Table 1: Various measured and calculated unit cell parameters under various stated conditions for NaT2 and, with respect to a three layer repeat, NaT3. The 2D map values are from experimental data while all other values are obtained from structure refinements. The $8 \times 1 \times 1$ and $4 \times 4 \times 4$ model R-factors include reflections comprising the 36 most intense resolved peaks. The $8 \times 1 \times 1$ model includes no flipped NaT2 molecules while the $4 \times 4 \times 4$ model includes 12 flipped NaT2 molecules (see text).

\begin{tabular}{llllllll}
\hline Model & $\mathrm{a}(\AA)$ & $\mathrm{b}(\AA)$ & $\mathrm{c}(\AA)$ & $\beta\left(^{\circ}\right)$ & $\mathrm{V}\left(\AA^{3}\right)$ & R-fac. & Temp \\
\hline NaT2 & & & & &. & & \\
644331.cif & $20.552(8)$ & $5.964(2)$ & $8.120(3)$ & $96.854(6)$ & $988.2(7)$ & 0.052 & $293 \mathrm{~K}$ \\
2D map & 20.436 & 5.943 & 8.120 & 96.92 & 979. & & Room \\
2D map & 20.491 & 5.947 & 8.131 & 96.38 & 985. & & $343 \mathrm{~K}$ \\
2D map & 20.486 & 5.961 & 8.148 & 96.12 & 992. & & $393 \mathrm{~K}$ \\
8x1x1 & 20.49 & 6.006 & 8.183 & 96.86 & 1000. & 0.114 & Room \\
8x1x1 & 20.62 & 6.054 & 8.292 & 96.46 & 1028. & 0.106 & $343 \mathrm{~K}$ \\
8x1x1 & 20.62 & 6.027 & 8.262 & 96.12 & 1021. & 0.098 & $393 \mathrm{~K}$ \\
4x4x4 & - & - & - & - & - & 0.092 & Room \\
\hline NaT3 & & & & & & & \\
644332.cif & $73.996(7)$ & $5.917(1)$ & $7.866(1)$ & $92.564(7)$ & $3440.4(5)$ & 0.145 & $187 \mathrm{~K}$ \\
6x2x2 & 76.44 & 5.843 & 7.942 & 101.0 & 3482. & & Room
\end{tabular}

The composite NaT3 GIXRD 2D map, at top in Fig. 4 is distinctly different. A major difference, vida infra, is that the observed peak positions and intensities differ noticeably from those of the reported bulk crystal. Moreover, in comparison to NaT2, there is considerably more arcing of the reflections along with broadening and more diffuse scattering along $q_{z}$ for the specific individual $k-\ell$ data sets (each of which forms a 1D line of scattering intensity 
perpendicular to the film surface in the $2 \mathrm{D}$ image). In addition there are weak but discernible localized scattering features along the ( $\left.h \begin{array}{ll}h & 0\end{array}\right)$ layer-line. These are symmetry forbidden in the conventional herringbone packing of NaT2 and NaT3 chains. As such this provides strong evidence that NaT3 thin films manifest a surface induced phase (or phases) with appreciable disorder.

Another feature that often appears in the NaT3 2D maps are the peaks at the $(h 00)$ positions. Technically these (h00) reflections should not be observed because of the intersection of the detector plane with the Ewald sphere. The aforementioned arcing cause weak vestiges of these peaks to appear in some 2D maps (not shown). We note that the nominal three layer repeat reported allows for measurable intensities in all (h00) reflections although the layer structure implies that $h=3 n$ peaks with $n=1,2,3, \ldots$ dominate. These NaT3 thin films only exhibit $h=3 n$ reflections along $q_{z}$ and, as compared to $(k l)$ layer-lines, have sharper peaks widths along $q_{z}$. Thus the cited three layer repeat of the bulk single crystals ${ }^{9}$ is absent in this SIP. Interestingly there is also an h00 scan from a thin film sample in Ref. 9 that also shows analogous characteristics.

GIXRD data from the NaT3 sample at the same two elevated temperatures, $343 \mathrm{~K}$ and $393 \mathrm{~K}$, are shown in a head-to-head comparisons of individual 1D $(k \ell)$ layer-line scans with those at room temperature in the supporting materials (series $\mathrm{H}$ ). Because this phase is more disordered the changes are less distinct than those in the NaT2 sample. Close inspection identifies variations in the peak positions comparable to those seen in NaT2.

\section{DFT and Preliminary MM Modeling}

As one might expect, the DFT calculations reproduce the intramolecular geometry relatively well thus validating the observed internal bond lengths and angles within both NaT2 and NaT3 molecules (see Supporting Materials). In NaT2 the largest deviations in the bond lengths reach approximately $0.03 \AA$ (in comparison with those obtained in the single crystal refinements) but most are less than $0.02 \AA$. For the MM calculations these bond length 
variations are generally within $0.03 \AA$ as well but some well known structural attributes cannot be reproduced. For example, the MM3 parameters cannot be configured to mimic the bond length variations within the napthyl rings without significant revisions. All told these small deviations are not expected to have a meaningful impact in the structural refinements that follow. ${ }^{16}$

The optimized DFT energies and unit cell dimensions are shown in Table 2 for the cases when the unit cell coordinates were locked and also when they were allowed to relax. Slightly different minimization algorithms were used in these two cases and so their respective energies cannot be directly compared. However comparisons of the conventional packing, Model A, and that corresponding to the situation where one oligomer is flipped, Model B, provide insight.

In NaT2 both models yield comparable ground-state energies and, as seen Fig. 5, only modest differences in their respective optimized unit cell geometries when the unit cell coordinates were free to move. The largest deviation, $0.26 \mathrm{eV}$ (per unit cell) larger in the case of Model B, corresponds to a difference of about $3 \mathrm{kcal} / \mathrm{mol}$ (on a per molecule basis) which is well in the range of acceptable values for justifying polymorphism. ${ }^{17}$

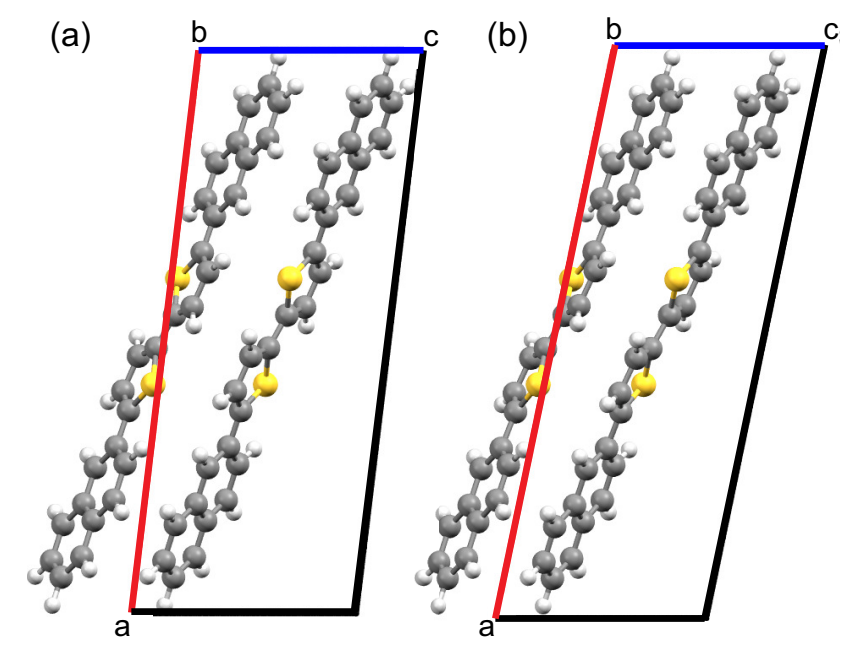

Figure 5: (a) Starting NaT2 configuration. (b) DFT optimized Model A NaT2 configuration.

Model A and B optimized unit cell parameters differ noticeably from that of the reported room temperature crystal structure. For example $\beta$ shifts by about $5^{\circ}$. Some of this is 
Table 2: Energy and lattice parameters of the optimized units cells. Fixed implies that the unit cell parameters were locked to those of the single crystal refinements of Ref. 9. In the case of NaT3 $a$ is one-third that of the real crystal.

\begin{tabular}{rrrrrrrrc} 
& Energy $[\mathrm{eV}]$ & $a[\AA]$ & $b[\AA]$ & $c[\AA]$ & $\alpha\left[^{\circ}\right]$ & $\beta\left[^{\circ}\right]$ & $\gamma\left[^{\circ}\right]$ & Volume $\left[\AA^{3}\right]$ \\
\hline $\begin{array}{c}\text { NaT2 Fixed } \\
\text { Model A }\end{array}$ & -1234.68 & 20.552 & 5.964 & 8.120 & 90.00 & 96.85 & 90.00 & 988.2 \\
Model B & -1234.50 & 20.552 & 5.964 & 8.120 & 90.00 & 96.85 & 90.00 & 988.2 \\
\hline $\begin{array}{c}\text { NaT2 Free } \\
\text { Model A }\end{array}$ & -1234.42 & 21.198 & 6.264 & 7.618 & 90.00 & 101.7 & 90.00 & 990.5 \\
Model B & -1234.16 & 21.670 & 6.464 & 7.303 & 89.19 & 103.4 & 90.64 & 995.0 \\
\hline NaT3 Fixed & & & & & & & & \\
Model A & -1455.46 & 24.665 & 5.917 & 7.850 & 90.00 & 92.56 & 90.00 & 1144 \\
Model B & -1455.40 & 24.665 & 5.917 & 7.850 & 90.00 & 92.56 & 90.00 & 1144 \\
\hline NaT3 Free & & & & & & & & \\
Model A & -1454.61 & 25.823 & 7.133 & 6.605 & 90.17 & 79.24 & 89.98 & 1195 \\
Model B & -1453.91 & 27.780 & 7.553 & 6.054 & 89.90 & 76.02 & 90.12 & 1233 \\
\hline
\end{tabular}

expected because the DFT is a ground state calculation and not at room temperature. However the unit cell volumes clearly reflect the fact that the intermolecular aspects of these DFT calculations are strictly qualitative in any absolute sense. Both ground state structures exhibit unit cell volumes larger than that of an NaT2 crystal at room temperature (and vdW bonded solids typically include rather sizable thermal expansion coefficients). There are limitations in the current DFT treatments of dispersion forces in organic compounds and so absolute accuracy is not expected.

The NaT3 DFT calculations are analogous. In the case of fixed unit cell coordinates the energies are essentially degenerate. However, when the unit cell parameters are freed, the variations become more extreme. Once again the DFT unit cell volumes are larger than those of the crystal. The $\beta$ angle shifts are over twice as large as those in the NaT2 calculations and the lattice constants, $a, b$, and $c$ vary considerably from that of reported in the single crystal study ${ }^{11}$ and from each other. The energy difference also is increased. If flipped molecules become incorporated within NaT3 crystals we theorize that these sites will manifest larger local strains and, as such, may be underlying driving force for the peculiar three layer repeat reported for bulk crystals and the disordered SIP we observe in thin films. 
We expect that these calculations are qualitative with respect to the actual unit cell parameters but have sufficient relative merit for comparing and justifying structural polymorphs. One point that should be reemphasized is that these are ground-state model calculations whereas all experimental studies are done at temperatures in excess of $295 \mathrm{~K}$.

\section{Structural Modeling and Refinement}

The first model calculation discussed, that of NaT2, employed an $8 \times 1 \times 1 \mathrm{SC}$ containing zero flipped molecules. To first order this is analogous to using a primitive unit cell but now the individual molecular positions and tilts within each layer become allowable free parameters. In this way the SC refinement can potentially identify attributes arising from local distortions. In general any increase in static disorder within the enlarged cell will be compensated by a decrease in the conventional crystallographic Debye scattering factors. Fig. 3 at bottom displays the simulated 2D GIXRD pattern and all key NaT2 features in the experimental data, to the right of the dashed yellow arc, are reproduced.

Figure 6 exhibits head-to-head comparisons of the individual experimental and refined model $k, \ell$ layers-lines. There are marked deviations, approaching factors of nearly three, in some of the relative peak intensities. Changing the intensity scale from linear (on left) to the square root (on right) better visualizes weaker features. Particularly troublesome are the peak intensities in the $\left(\begin{array}{lll}h & 1 & 0\end{array}\right)$ and $\left(\begin{array}{lll}h & 2 & 1\end{array}\right)$ layer-lines. Exact fits to the lineshapes are also challenging. There is a very noticeable asymmetry with a larger wing appearing on the low $q_{z}$ side. This may be attributed to anisotropy in the crystal domain sizes and lattice spacings in the direction perpendicular to $q_{z}$ Still the final calculated $R$-factors, 0.072 (with 20 peaks) and 0.114 (with 36 peaks, Table 3 on page S3 in the supporting materials, SM), are quite good but somewhat larger than that reported in the single crystal study ${ }^{9}$ (with an $\mathrm{R}=0.05)$. Direct comparisons can be misleading because significantly more reflections are accessed in conventional single crystal refinements.

Once local $180^{\circ}$ flips, about the long molecular axis, are introduced any subsequent SRs 

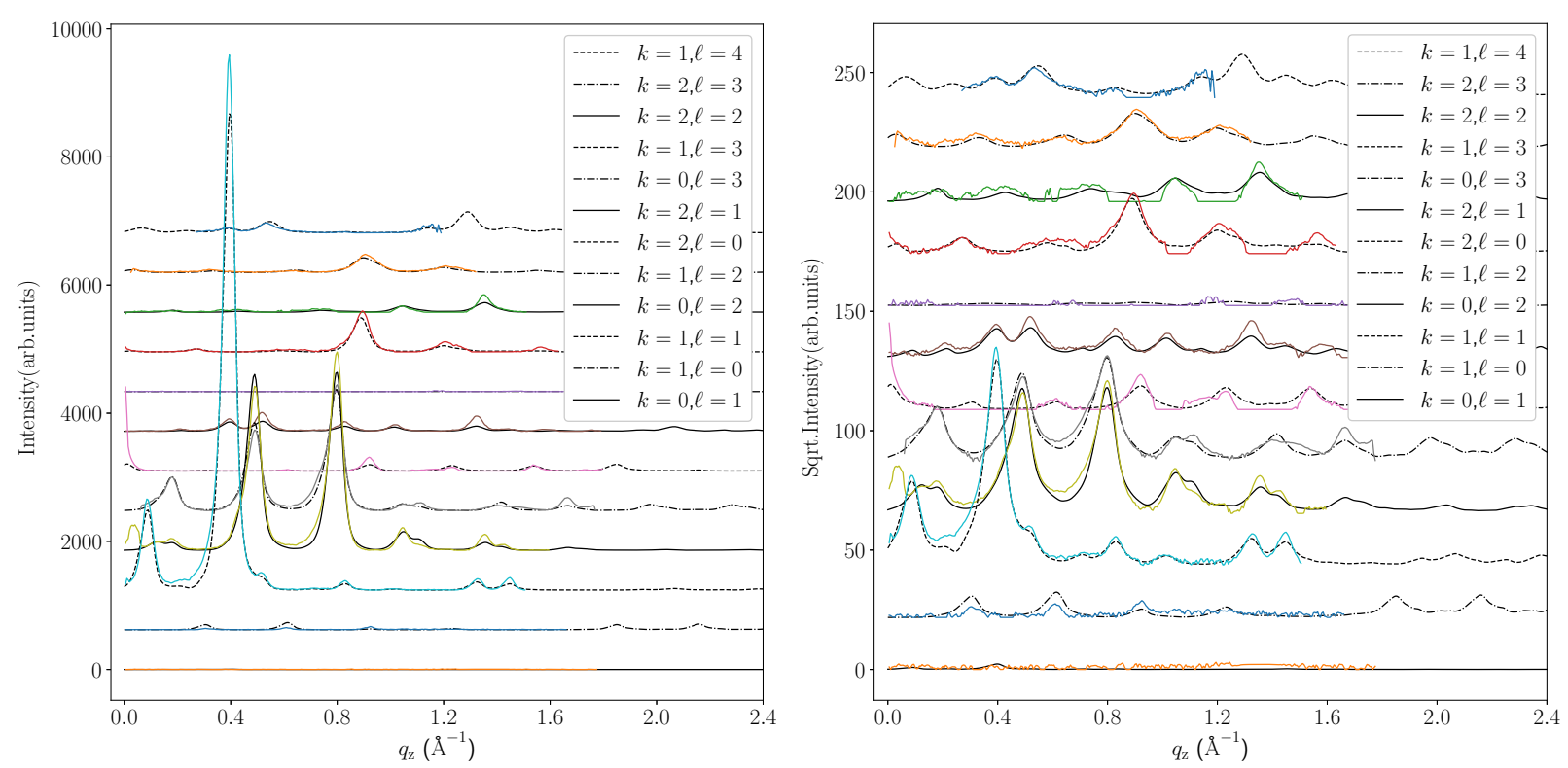

Figure 6: Comparisons, at left linear and at right square root, of calculated (black) and experimental (color) intensities along various $k l$ "layer-lines" of the NaT2 $8 \times 1 \times 1$ "no flip" model at room temperature.

necessarily become more complicated because of the local relaxation needed to accommodate these defects. Although MM calculations have clear limitations, a preliminary MM minimum energy calculation enables the system to relax locally to more energetically favorable starting configurations.

In Fig. 7 (and series A, pages S4-S6, of the SM) we present the results of a SR using a model in which eight monomer flips have been chosen at random within each $4 \times 4 \times 4$ supercell (and initially relaxed via MM). This refinement once again assumes rigid molecules which, within individual $4 \times 4$ layers, are permitted to reorient and translate. We find that the overall displacements are generally small and do not yield significant increases in the MM energies. The $R$-factor for this and related models including local disorder are slightly less than that in the "no flip" model above. In this case $\mathrm{R}=0.058$ (using 20 peaks) or 0.088 (with 36 peaks). The improvement is modest but does not appreciably address the problems with the ( $\left.h \begin{array}{lll} & 1 & 0\end{array}\right)$ and ( $\left.h \begin{array}{lll}h & 1\end{array}\right)$ intensities. Increasing the number of random flips to twelve (series B, pages S7-S9, of the SM) yields comparable fits with $\mathrm{R}=0.057$ (using 20 peaks) 
or 0.091 (with 36 peaks). In both these cases the changes are rather subtle to discern and highlight how difficult it may be to assess local disorder in these and related compounds.

Although the aforementioned systematic failings are not satisfactorily resolved, this analysis clearly demonstrates that a high degree of structural disorder can be accommodated within the crystal interior in addition to the extensive disorder that occurs at the periphery of crystalites at grain boundaries. To our knowledge this issue has not been assessed by rigorous modeling methods in prior publications. This finding has broad significance because of the implications this attribute may have in limiting OE device performance characteristics.

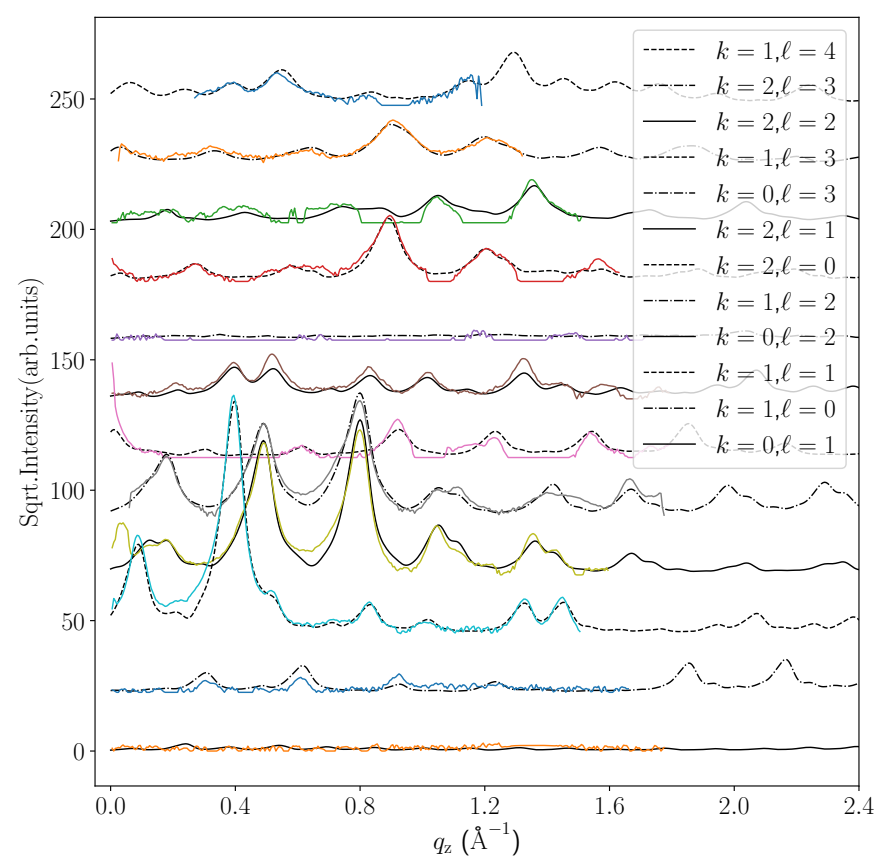

Figure 7: Comparison of calculated (black) and experimental (color) intensities along various $k l$ "layer-lines" of the NaT2 $4 \times 4 \times 4$ model (incorporating eight monomer flips) at room temperature.

Substrate interactions in combination with an increased proportion of surface interfaces may cause thin film lattices to deviate from those of the bulk. The room temperature lattice constants of the NaT2 thin film, as determined from a position analysis of the most intense peaks in the $2 \mathrm{D}$ maps, are close to those of the single crystal ${ }^{10}$ with the largest deviation that of the $a$-axis at about $0.5 \%$. The lattice constants deduced from refinement of an $8 \times 1 \times 1$ supercell model are not identical those values derived directly from the 2D maps but the 
largest deviation, for the value of $b$-axis repeat, is only about $0.7 \%$.

Both the NaT2 and NaT3 thin films are fabricated in a non-equilibrium process and so even mild thermal treatment may lead to more ordered structures if the local energy barriers are small. Although increasing temperature generally results in a small increase in the volume of the unit cell, as one may expect in a vdW bonded solid, any other changes were minimal. The most significant observed difference is a monotonic decrease in the $\beta$ angle of approximately $0.7^{\circ}$ with the $120 \mathrm{~K}$ increase in the film temperature.

As far as 2D GIXRD patterns are concerned there are small scale systematic intensity changes and peak position shifts consistent with variations in the lattice constants and slightly increased thermal motions. The calculated R-factors from analogous fits to the data at $343 \mathrm{~K}$ and $393 \mathrm{~K}$ do not vary appreciably (sets C and D, pages S10-S15, in the SM) from those at room temperature.

We now turn to the NaT3 structural analysis. Modeling the NaT3 thin film structure is necessarily more complicated for at least two reasons. Firstly, the molecule itself is slightly larger with an additional seven atoms from the third thiophene ring. Thus there is a greater likelihood of closely related local energy minima in the molecular packing. Secondly, the reported single crystal unit cell has a nominal repeat consisting of three molecular layers. Using the reported crystallographic .cif file ${ }^{11}$ as a starting point we address the existence of two symmetry related molecular orientations within the middle layer by constructing a six layer (i.e., 12 molecules) facsimile and then optimizing this structure by an MM energy minimization. The structure function for this model yields peak intensities and positions in reasonably good agreement with those obtained in the reported single crystal NaT3 structure.

The simulated 2D image (E1 on page S16 of the SM) does not well match that of the experimental thin film data. This misfit is better seen in a direct comparison of the various resolved layer lines as shown in E2, page S16, of the SM. Neither the peak positions nor the general scatting intensities are in reasonable agreement. As such this unambiguously supports the assertion that NaT3 thin films manifest a SIP. There are at least two reason- 
able starting points for addressing the structure within the disordered SIP of NaT3. One possibility is that the nominal single crystal structure still persists in the thin films but it has a distorted unit cell lattice which includes increased appreciable disorder. Alternately one could look at the motif as observed in NaT2 thin films and then incorporate additional disorder. Of these two possibilities the modeling indicates that the latter structural ordering is more likely. Starting with the NaT3 single crystal model just discussed, then setting $\beta=94.76^{\circ}$ and increasing the $a$-axis repeat by $0.3 \%$ and thereafter employing a MM optimization yields a scattering pattern shown in Fig. 8 at left. There is exceptionally good agreement with respect to the features in the ( $\left.h \begin{array}{ll}h & 1\end{array}\right)$ layer line but, unfortunately, this model fails badly just about everywhere else. We also reiterate that there are no $(h 00)$ reflections consistent with anything but a nominal single layer repeat.
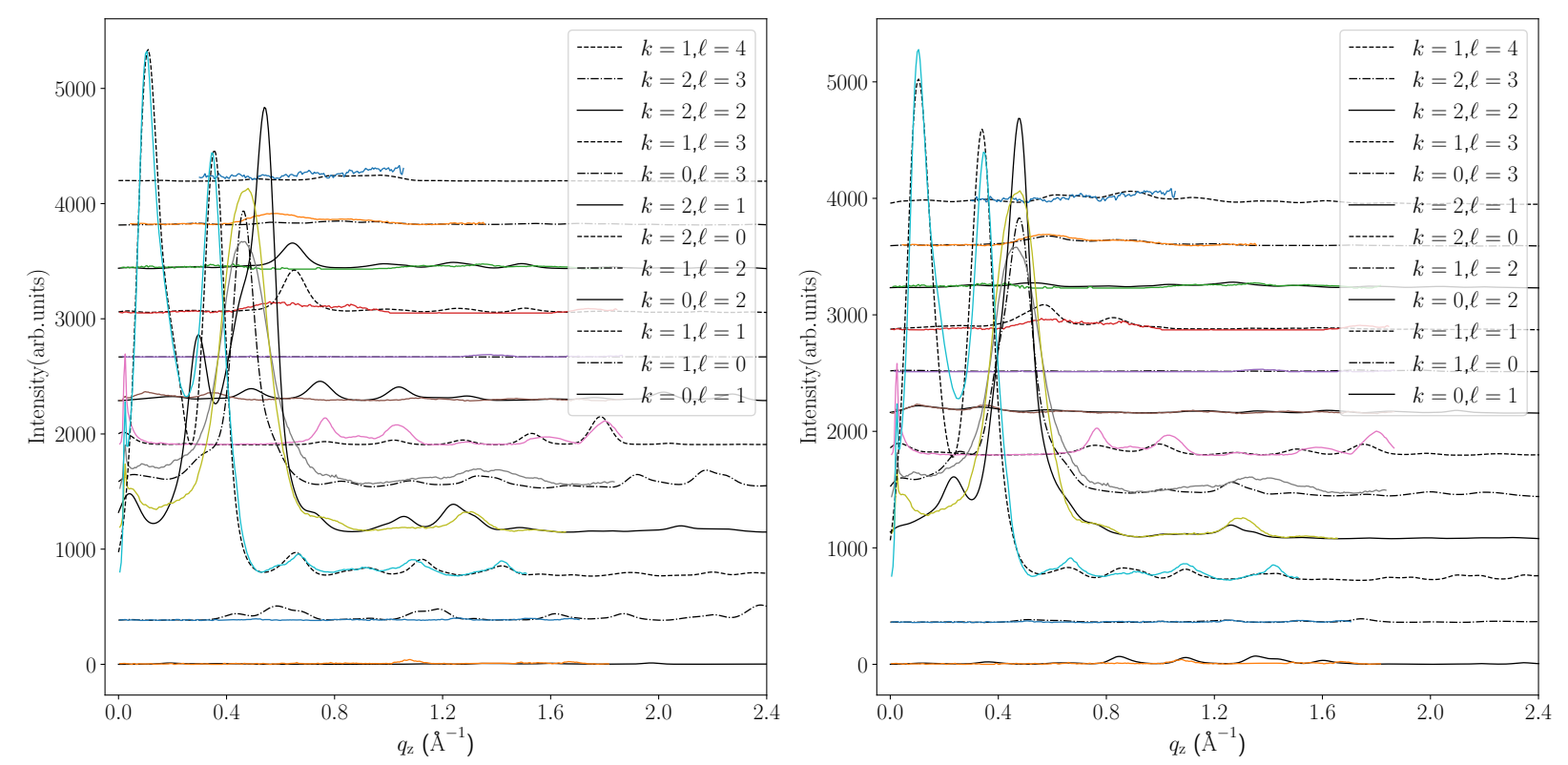

Figure 8: Left: Comparison of calculated (black, for a model approximating the single crystal structure with $\beta=94.76^{\circ}$ ) and experimental (color) intensities along various $k l$ "layer-lines" for an NaT3 thin film. Right: Comparison of calculated (black, for the NaT3 model discussed in the text (with $\beta=100.96^{\circ}$ ) and experimental (color) intensities along various $k l$ "layer-lines" for an NaT3 thin film

The alternate approach, based on the single layer repeat seen in NaT2 and then introducing a large number of flipped molecules, does much better but still has shortcomings. 
The simulated 2D scattering pattern, shown at bottom in Fig. 3, strongly resembles that of the experimental data with respect to the strongest scattering features. However direct comparison of the individual layer lines as seen in Fig. 8 at right shows clear disagreement in places. For example there is relative poor agreement for peaks forming the $(h 20)$ layer line. A large number of related models were tested without notable improvement. It is likely that a SC containing just 48 individual molecules may be just too limited in scope to adequately mimic this SIP. Subsequent molecular dynamics studies at finite temperatures may provide better insight.

\section{Conclusions}

The presence of structural disorder and its effects on electron transport within organic hosts are issues which have seen much effort especially in recent theoretical studies. ${ }^{18,19}$ Computational methods have advanced so that sufficiently small molecules or clusters can be assessed with ab initio methods whereas larger systems necessarily rely on calculations based on various empirical force fields. ${ }^{20}$ This disorder can be both static, as considered here, or dynamic, from finite temperature effects. Depending the specific nature of the material this disorder can be responsible for either increasing or decreasing charge carrier mobilities. ${ }^{21}$ From the perspective of electronic or optical properties, which typically transpire at nanosecond or shorter time scales, dynamic disorder has almost a static appearance because of this temporal disparity.

As far as NaT2 thin films go, we infer that its charge mobility will almost certainly be impacted by the presence of the flipped molecules. Without follow up calculations of the transport we can only speculate. If these molecules leads to poorer transport then increasing crystallite size, which is often suggested as a method for enhancing charge transport, may not be an especially useful strategy. ${ }^{14}$ It is interesting to note that NaT3 thin-film OFETs, which clearly incorporate greater structural disorder than NaT2, typically exhibit higher 
mobilities ${ }^{9}$ but are still approximately a factor of ten less than that of NaT4. ${ }^{22}$

On the other hand it would be of great utility if, in the molecular design of small molecule OFETs, to develop materials which could be rendered insensitive to this type of disorder. Recent efforts, e.g. pentacene, ${ }^{23}$ seek to more fully relate thermal and transport properties by both experimental and computational methods. Of course pentacene, which has bilateral symmetry along its long molecular axis, will be insensitive to these $180^{\circ}$ flips.

Deciphering the molecular level structure and its relationship to electronic or optical properties within organic thin-films remains a challenging research topic ${ }^{24,25}$ and, despite intense efforts over many decades, important questions remain. From a structural perspective one desires a full quantitative agreement between the experimental results and model structures. ${ }^{26,27}$ This study of NaT2 thin-films supports the assertion that appreciable structural disorder is present within the interior of crystalline regions. The SIP that typifies thin-film NaT3 samples exemplifies the strong sensitivity of structural phase behavior to the material and environment. While both models achieve reasonably good fits to the experimental data there are systemic shortcomings whose exact origin remains a mystery. Quantitative agreement is an enviable goal but, as is often true in complicated systems, questions of uniqueness will arise.

\section{Experimental}

The experimental OFET devices used in this work and film characteristics have been reported previously. ${ }^{13-15}$ NaT2 and NaT3 have many similarities. Phenomenologically, these films exhibit a plate like crystal habit when grown on $\mathrm{SiO}_{2}$. The top surface of the films exhibits a surface roughness ranging from 6 to $8 \mathrm{~nm}$ in the thinner films and from 2 to $4 \mathrm{~nm}$ in thicker films. On occasion fibrous crystals have been obtained. Ref. 14 reports that the film morphology differs near the substrate interface and, after some two to four molecular layers, it then adopts the NaTx end-on layering for the remainder of the films thickness 
which ranges from 20 to $40 \mathrm{~nm}$.

The basic OFET structure employs an $n$-doped Si substrate with a $200 \mathrm{~nm}$ thick $\mathrm{SiO}_{2}$ gate dielectric and thin interdigitated $\mathrm{Ti} / \mathrm{Au}$ e-beam fabricated electrodes. Oligomer synthesis used a Stille cross-coupling method outlined in Ref. 28. All NaTx thin films were deposited at room temperature using vacuum sublimation (typically $10^{-8}$ mbar with a deposition rate of ca. $0.1 \AA / \mathrm{s}$ ) to film thicknesses that varied from 50 to $200 \mathrm{~nm}$. In these multilayer stacks the GIXRD is dominated by scattering from NaTx molecules standing on end with the molecular axes nearly perpendicular to the substrate surface.

Detailed investion

GIXRD measurements were performed at the UK CRG beamline XMaS (BM28) at the European Synchrotron Radiation Facility (ESRF) inside a enclosed chamber filled with helium gas in order to reduce beam absorption and air scattering. Ten keV X-rays were used to form a nominal footprint of $50 \mu \mathrm{m} \times 350 \mu \mathrm{m}$ (height $\times$ width). The specific angle of incidence was optimized for maximum sample scattering intensity (and kept between the critical angles of $\mathrm{NaTx}_{\mathrm{T}}$ and $\mathrm{SiO}_{2}$ of $0.141^{\circ}$ and $0.173^{\circ}$ respectively). The $2 \mathrm{D}$ intensity images were recorded with an MAR165 CCD detector. Two different detector arrangements were employed: 1. A forward direction scan with a sample-to-detector distance of $338 \mathrm{~mm}$ (covering a horizontal $q$-vector range (parallel to the surface) of $q_{x y}<1.95 \AA^{-1}$ and vertically (perpendicular to the surface) up to $q_{z}<1.5 \AA^{-1}$ and 2. A second forward scan with $q_{x y}<3.5 \AA^{-1}$. These two scans were then scaled and merged to give a single GIXRD 2D image. All 2D maps contained two radially sharp and intermittent rings at relatively high angles corresponding to the scattering from the (111) and (200) reflections of the gold electrodes. Using these reflections as calibration measurements sample-to-detector distances were derived for the angle setup. The raw pixel data were transformed into reciprocal $q$ space as described in Ref. 29.

DFT ground-state calculations were used for assessing the relative energies of unit cell structures containing two NaT2 or NaT3 molecules. All DFT computations reported here 
employ the Atomic Simulation Environment ${ }^{30}$ and the GPAW package ${ }^{31,32}$ (PAW for projectoraugmented wave method) in combination with meta Generalized Gradient Approximation xc-functionals and periodic boundary conditions. These functionals can be used to model intramolecular covalent bonds.

To account for the weaker long-range interactions a number of functionals were tested. Ultimately the system was modeled with the mBEEF-vdW (meta Bayesian Error Estimationvan der Waals) exchange functional. The mBEEF-vdW xc-functional has been shown to have reasonably good accuracy ${ }^{33}$ when estimating lattice constants.

All DFT calculations used a plane wave cut-off energy of $700 \mathrm{eV}$ and Monkhorst-Pack $(1 \times 6 \times 4) k$-point grid. Two different scenarios were examined, one with the lattice locked to the unit cell parameters from Ref. 9 (for NaT3 that implies $a / 3$ ) and the other allowing the unit cells to relax. System relaxation allowed for simultaneous movement of the atoms and the unit cell. The system was considered relaxed when the total force on each atom was less than $0.05 \mathrm{eV} / \AA$. The $k$-point grid was chosen to satisfy $k_{1,2,3} \mathbf{r}_{1,2,3}>30$ except for the $a$ lattice repeat (due to its large size). Because of computational time constraints a $k$-point convergence test was only carried out with locked unit cell parameters.

The MM calculations employed the Tinker package ${ }^{34}$ using well known ${ }^{35}$ but here slightly modified MM3 force fields (i.e., MM3*). The suitability and transferability ${ }^{36}$ of empirical force fields, specifically MM3, ${ }^{37}$ is important. In our case there were two primary justifications for these ad hoc alterations. First, we wished to obtain specific carbon-carbon and carbon-sulfur bond lengths and angles that more closely match those ascertained in the converged DFT calculations and from single crystal refinements. ${ }^{9}$ We presumed that this would reduce overt systematic errors in the calculated intensities of the subsequent SF calculations. The various bond lengths are reported in Tables 1 and 2 of the supplementary materials (SM, on S1 and S2). A second modification was small ad-hoc changes in the intermolecular pair potential Lennard-Jones (LJ) coefficients. Empirical force field expressions are straightforward to use but they, in reality, reflect rather simplistic approximations of the true pair 
interactions. Preliminary NaT2 MM (and molecular dynamics calculations as well which are not discussed in this work) yielded equilibrium structures somewhat different than those of the single crystal refinement. Particularly troublesome was the appearance of two distinct energy minima with different unit cell lattice constants and oligomer tilts. With standard MM3 parameters the one lowest in energy had unit cell parameters in poorer agreement with those observed in the single crystal study. ${ }^{9}$ Small changes in the LJ carbon and sulfur potential well depth coefficients (typically as $\epsilon$ ) lowered the relative energy of the minima which most closely matched the unit cell parameters obtained in the NaT2 single crystal refinement.

The utility of the MM methodology becomes evident in models which include disorder (i.e., flipped NaTx molecules). The two closely related polymorphic structures of interest are shown for NaT2 in Fig. 2. Here the letters U and D (for "up" and "down" respectively) with associated red pointing arrows are used to distinguish the pair. The only difference is that one of the two oligomers comprising the unit cell is flipped $180^{\circ}$ about its long axis and reflects a type of orientational disorder. Not surprisingly MM and DFT calculations find that the unit cells of the two cited polymorphs have slightly different equilibrium structures (vide infra). Thus when incorporating flipped oligomers into any SC necessitates structural relaxation locally to accommodate this defect and thereby obtain a more reasonable starting structure for use in subsequent SF refinements. SC models containing upwards of $25 \%$ of units cells with defects were examined.

Once an optimized MM model was formed the resulting base structure was exported for use in a custom SF refinement. Adjustable parameters includes those of the unit cell plus oligomer orientation and translation (uniformly for the herringbone pairs within each layer). In general the geometry within an individual oligomer was keep rigid so as to limit the number of free parameters. SF intensities for the full grid of reciprocal lattice points were calculated and then projected onto the Ewald surface intersected by the 2D detector following methods well described in Ref. 38. Standard geometrical corrections (i.e., Lorentz and polarization), 
multiplicity, Deybe thermal factors were all incorporated in the SF calculations. In addition the carbon-hydrogen bond length was shortened approximately $0.25 \AA$ when transferring the MM generated coordinates for use in the SF calculations (an artifact of X-ray scattering which only probes the electron wavefunction). We did not observe scattering signatures indicative of multiple scattering. ${ }^{39}$ The $a^{*}$ reprocal lattice $(\mathrm{RL})$ vector is perpendicular to the substrate but there is cylindrical powder averaging of the RL points about this axis to give a uniplanar ${ }^{40}$ type texture. Additionally there can be arcing and broadening of the RL points due to crystallite finite size distribution and paracrystallinity. These effects were mimicked through a limited set of Gaussian and/or composite Gaussian/Lorentzian functions that were convoluted with the original intensities at each Miller index. These also could be chosen as fixed or constrained free parameters in the SRs. The grid point spacing in the simulation scattering patterns was typically $0.01 \AA^{-1}$ in both the $q_{x y}$ and $q_{z}$ directions.

To facilitate direct quantitative comparisons of the simulated 2D GIXRD maps with the experimental data both images were transformed into a series of 1D profiles for every observed $k, \ell \neq 0,0$ "layer line" ${ }^{41}$ by integrating data in appropriate $q_{x y}$ ranges (typically between $q_{x y}-0.03 \AA^{-1}$ and $\left.q_{x y}+0.05 \AA^{-1}\right)$. These 1D profiles represent scattering intensity along the $a^{*}$ direction (i.e., perpendicular to the film surface). Up to twelve individual sets of $1 \mathrm{D}$ data were used in the SRs. The SRs function minimized was a weighted $\chi^{2}$ of the difference between experimental and calculated layer-lines. The weighting scheme eliminated positions with scattering artifacts and decreased the weighting when the relative scattering intensities were lower. An advantage of this approach was that it allowed for a straightforward consideration of the $q_{z}$ lineshapes and closely spaced reflections. To enable comparisons by more conventional methods (i.e., an $R$-factor) the specific position of individual reflections were determined then a conventional unweighted $R$-factor was calculated using

$$
R=\left[\sum|| F_{\text {expt }}|-| F_{\text {calc }}||\right] / \sum\left|F_{\text {expt }}\right|
$$


and tabulated. If there are overlapping peaks only the most intense reflection has been identified in the tables. As a starting point the structure of the published NaT2 unit cell was used and our calculated intensities were consistent with those generated with those obtained using standard crystallographic software.

\section{Acknowledgement}

The authors are grateful for financial support from the Otto Mønsteds Fond (grant no: 1612-0653) and DANSCATT (grant no: 7055-00005B) as well as for Nordea Fonden's Residence Programme. We thank also Andreas Osadnik and Arne Lützen of the University of Bonn for providing oligomer powders, Oier Bikondoa of the ESRF for experimental assistance and Denis Andrienko and Peregrine Ross Warren of the University of Oxford and Martin Meedom Nielsen of Technical University of Denmark for discussions. All DFT calculations were performed at the Niflheim cluster at Technical University of Denmark.

\section{Supporting Information Available}

Included in the supporting information are comparisons of the intramolecular bond lengths and the thiophene $\mathrm{C}-\mathrm{S}-\mathrm{C}$ bond angles. In addition there are numerous simulated NaT2 GIXRD patterns, their comparisons with the experimental data and the explicit Miller indicies used for assessing the various quoted R-factors. Finally there are head-to-head comparison of the $k \ell$ "layer lines" at room temperature and two elevated temperatures for both NaT2 and NaT3.

Tables 1 (NaT2 on S1) and 2 (NaT3 on S2): Comparisons of selected bond lengths and angles.

Table 3 (on page S3): Comparisons of calculated and experimental structure factors for various $k l$ layer-lines for NaT2 room temperature data and the $8 \times 1 \times 1$ supercell model (no NaT2 molecule flips). 
A1 to F1: Simulated 2D grazing incidence patterns.

A2 to F2: Comparisons of calculated and experimental intensities for various $k l$ layer-lines. A3 to D3: As above but using the square root of the intensities.

A4 to D4: Tables comparing the calculated and measured structure factors of selected crystal reflections

\begin{tabular}{ccccccl}
\hline Series & Monomer & Temperature & Supercell & Monomer Flips & Pages & Note \\
\hline A & NaT2 & Room & $4 \times 4 \times 4$ & 8 & S4-S6 & \\
B & NaT2 & Room & $4 \times 4 \times 4$ & 12 & S7-S9 & \\
C & NaT2 & $70{ }^{\circ} \mathrm{C}$ & $8 \times 1 \times 1$ & None & S10-S12 & \\
D & NaT2 & $120{ }^{\circ} \mathrm{C}$ & $8 \times 1 \times 1$ & None & S13-S15 & \\
E & NaT3 & Room & $6 \times 1 \times 1$ & None & S16 & $\begin{array}{l}\text { Approximates single } \\
\text { crystal structure }\end{array}$ \\
F & NaT3 & Room & $6 \times 2 \times 2$ & Included & S17 & Best fit \\
\hline
\end{tabular}

Figures G1-G3 on pages S18-S20: Layer-line intensities $(\sqrt{I})$ for the NaT2 sample at room temperature, 343K, and 393K.

Figures H1-H3 on pages S21-S23: Layer-line intensities $(\sqrt{I})$ for the NaT3 sample at room temperature, $343 \mathrm{~K}$, and 393K. 


\section{References}

(1) Dimitrakopoulos, C.; Malenfant, P. Organic Thin Film Transistors for Large Area Electronics. Adv. Mater. 2002, 14, 99-117.

(2) Klauk, H. Organic thin-film transistors. Chem. Soc. Rev. 2010, 39, 2643-2666.

(3) Torsi, L.; Magliulo, M.; Manoli, K.; Palazzo, G. Organic field-effect transistor sensors: a tutorial review. Chem. Soc. Rev. 2013, 42, 8612-8628.

(4) Sirringhaus, H. 25th Anniversary Article: Organic Field-Effect Transistors: The Path Beyond Amorphous Silicon. Adv. Mater. 2014, 26, 1319-1335.

(5) Li, Y.; Wan, J.; Smilgies, D.-M.; Bouffard, N.; Sun, R.; Headrick, R. L. Nucleation and strain-stabilization during organic semiconductor thin film deposition. Sci. Rep. 2016, 6,32620 .

(6) Jones, A. O. F.; Chattopadhyay, B.; Geerts, Y. H.; Resel, R. Substrate-Induced and Thin-Film Phases: Polymorphism of Organic Materials on Surfaces. Adv. Funct. Mater. 2016, 26, 2233-2255.

(7) Resel, R.; Jones, A. O. F.; Schweicher, G.; Fischer, R.; Demitri, N.; Geerts, Y. H. Polymorphism of terthiophene with surface confinement. IUCrJ 2018, 5, 304-308.

(8) Liscio, F.; Ferlauto, L.; Matta, M.; Pfattner, R.; Murgia, M.; Rovira, C.; MasTorrent, M.; Zerbetto, F.; Milita, S.; Biscarini, F. Changes of the Molecular Structure in Organic Thin Film Transistors during Operation. J. Phys. Chem. C 2015, 119, $15912-15918$.

(9) Tian, H. K.; Shi, J. W.; He, B.; Hu, N. H.; Dong, S. Q.; Yan, D. H.; Zhang, J. P.; Geng, Y. H.; Wang, F. S. Naphthyl and Thionaphthyl End-Capped Oligothiophenes as Organic Semiconductors: Effect of Chain Length and End-Capping Groups. Adv. Funct. Mater. 2007, 17, 1940-1951. 
(10) 644331.cif in the Cambridge Structural Database.

(11) 644332.cif in the Cambridge Structural Database.

(12) Salzmann, I.; Nabok, D.; Oehzelt, M.; Duhm, S.; Moser, A.; Heimel, G.; Puschnig, P.; Ambrosch-Draxl, C.; Rabe, J. P.; Koch, N. Structure Solution of the 6,13-Pentacenequinone Surface-Induced Polymorph by Combining X-ray Diffraction Reciprocal-Space Mapping and Theoretical Structure Modeling. Cryst. Growth Des. 2011, 11, 600-606.

(13) Huss-Hansen, M. K.; Lauritzen, A. E.; Bikondoa, O.; Torkkeli, M.; Tavares, L.; Knaapila, M.; Kjelstrup-Hansen, J. Structural stability of naphthyl end-capped oligothiophenes in organic field-effect transistors measured by grazing-incidence X-ray diffraction in operando. Org. Elect. 2017, 49, 375-381.

(14) Lauritzen, A. E.; Torkkeli, M.; Bikondoa, O.; Linnet, J.; Tavares, L.; KjelstrupHansen, J.; Knaapila, M. Structural Evaluation of 5,5'-Bis(naphth-2-yl)-2,2'bithiophene in Organic Field-Effect Transistors with $n$-Octadecyltrichlorosilane Coated SiO2 Gate Dielectric. Langmuir 2018, 34, 6727-6736.

(15) Huss-Hansen, M. K.; Hansteen, M.; Linnet, J.; Walther, A. R.; Kjelstrup-Hansen, J.; Knaapila, M. Structural basis for a naphthyl end-capped oligothiophene with embedded metallic nanoparticles for organic field-effect transistors. Applied Physics Letters 2018, $113,251903$.

(16) One small systemic effect is in regards to the carbon-hydrogen bond length which is systematically underestimated in XRD studies. To compensate for that the nominal $1.1 \AA \mathrm{C}-\mathrm{H}$ bond length in the MM derived parameters is set to $0.85 \AA$ prior to structure factor calculations.

(17) Nangia, A. Conformational Polymorphism in Organic Crystals. Acc. Chem. Res. 2008, 41, 595-604. 
(18) Habgood, M.; Grau-Crespo, R.; Price, S. L. Substitutional and orientational disorder in organic crystals: a symmetry-adapted ensemble model. Phys. Chem. Chem. Phys. 2011, 13, 9590-9600.

(19) Yavuz, I.; Martin, B. N.; Park, J.; Houk, K. N. Theoretical Study of the Molecular Ordering, Paracrystallinity, And Charge Mobilities of Oligomers in Different Crystalline Phases. J. Am. Chem. Soc. 2015, 137, 2856-2866.

(20) Casalegno, M.; Moret, M.; Resel, R.; Raos, G. Surface Reconstructions in Organic Crystals: Simulations of the Effect of Temperature and Defectivity on Bulk and (001) Surfaces of 2, 2' : 6', 2"-Ternaphthalene. Crystal Growth \& Design 2016, 16, 412-422.

(21) Vehoff, T.; Baumeier, B.; Troisi, A.; Andrienko, D. Charge Transport in Organic Crystals: Role of Disorder and Topological Connectivity. J. Am. Chem. Soc. 2010, 132, $11702-11708$.

(22) Tian, H. K.; Shi, J. W.; Yan, D. H.; Wang, L. X.; Geng, Y. H.; Wang, F. S. Naphthyl End-Capped Quarterthiophene: A Simple Organic Semiconductor with High Mobility and Air Stability. Adv. Mater. 2006, 18, 2149-2152.

(23) Ando, M.; Yoneya, M.; Ishii, H.; Minakata, T.; Kawasaki, M.; Duffy, C. M.; Phillips, R.; Sirringhaus, H. Disorder and localization dynamics in polymorphs of the molecular semiconductor pentacene probed by in situ micro-Raman spectroscopy and molecular dynamics simulations. Phys. Rev. Mater. 2019, 3, 025601.

(24) Atahan-Evrenk, S.; Aspuru-Guzik, A. Prediction and Theoretical Characterization of $p$ Type Organic Semiconductor Crystals for Field-Effect Transistor Applications. Topics in Current Chemistry 2014, 345.

(25) Jones, A. O. F.; Röthel, C.; Lassnig, R.; Bedoya-Martínez, O. N.; Christian, P.; Salzmann, I.; Kunert, B.; Winkler, A.; Resel, R. Solution of an elusive pigment crystal 
structure from a thin film: a combined X-ray diffraction and computational study. CrystEngComm 2017, 19, 1902-1911.

(26) Lemaur, V.; Bouzakraoui, S.; Olivier, Y.; Brocorens, P.; Burhin, C.; El Beghdadi, J.; Martin-Hoyas, A.; Jonas, A. M.; Serban, D. A.; Vlad, A.; Boucher, N.; Leroy, J.; Sferrazza, M.; Mouthuy, P.-O.; Melinte, S.; Sergeev, S.; Geerts, Y.; Lazzaroni, R.; Cornil, J.; Nysten, B. Structural and Charge-Transport Properties of a Liquid-Crystalline Structural and Charge-Transport Properties of a Liquid-Crystalline $\alpha, \omega$-Disubstituted Thiophene Derivative: A Joint Experimental and Theoretical Study. J. Phys. Chem. C 2010, 114, 4617-4627.

(27) Gbabode, G.; Dohr, M.; Niebel, C.; Balandier, J.-Y.; Ruzié, C.; Négrier, P.; Mondieig, D.; Geerts, Y. H.; Resel, R.; Sferrazza, M. X-ray Structural Investigation of Nonsymmetrically and Symmetrically Alkylated [1]Benzothieno[3,2-b]benzothiophene Derivatives in Bulk and Thin Films. ACS Appl. Mater. Interfaces 2014, 6, 1341313421.

(28) Liu, X.; Wallmann, I.; Boudinov, H.; Kjelstrup-Hansen, J.; Schiek, M.; Lützen, A.; Rubahn, H.-G. AC-biased organic light-emitting field-effect transistors from naphthyl end-capped oligothiophenes. Org. Elect. 2010, 11, 1096 - 1102.

(29) Schlepütz, C. M.; Mariager, S. O.; Pauli, S. A.; Feidenhans'l, R.; Willmott, P. R. Angle calculations for a (2+3)-type diffractometer: focus on area detectors. J. Appl. Cryst. 2011, 44, 73-83.

(30) Larsen, A. H.; Mortensen, J. J.; Blomqvist, J.; Castelli, I. E.; Christensen, R.; Dułak, M.; Friis, J.; Groves, M. N.; Hammer, B.; Hargus, C.; Hermes, E. D.; Jennings, P. C.; Jensen, P. B.; Kermode, J.; Kitchin, J. R.; Kolsbjerg, E. L.; Kubal, J.; Kaasbjerg, K.; Lysgaard, S.; Maronsson, J. B.; Maxson, T.; Olsen, T.; Pastewka, L.; Peterson, A.; Rostgaard, C.; Schiøtz, J.; Schütt, O.; Strange, M.; Thygesen, K. S.; 
Vegge, T.; Vilhelmsen, L.; Walter, M.; Zeng, Z.; Jacobsen, K. W. The atomic simulation environment - a Python library for working with atoms. J. Phys. Condens. Matter 2017, 29, 273002.

(31) Mortensen, J. J.; Hansen, L. B.; Jacobsen, K. W. Real space grid implementation of the projector augmented wave method. Phys. Rev. B 2005, 71, 035109.

(32) Enkovaara, J.; Rostgaard, C.; Mortensen, J. J.; Chen, J.; Dułak, M.; Ferrighi, L.; Gavnholt, J.; Glinsvad, C.; Haikola, V.; Hansen, H. A.; Kristoffersen, H. H.; Kuisma, M.; Larsen, A. H.; Lehtovaara, L.; Ljungberg, M.; LopezAcevedo, O.; Moses, P. G.; Ojanen, J.; Olsen, T.; Petzold, V.; Romero, N. A.; Stausholm-Møller, J.; Strange, M.; Tritsaris, G. A.; Vanin, M.; Walter, M.; Hammer, B.; Häkkinen, H.; Madsen, G. K. H.; Nieminen, R. M.; Nørskov, J. K.; Puska, M.; Rantala, T. T.; Schiøtz, J.; Thygesen, K. S.; Jacobsen, K. W. Electronic structure calculations with GPAW: a real-space implementation of the projector augmented-wave method. J. Phys. Condens. Matter 2010, 22, 253202.

(33) Lundgaard, K. T.; Wellendorff, J.; Voss, J.; Jacobsen, K. W.; Bligaard, T. mBEEFvdW: Robust fitting of error estimation density functionals. Phys. Rev. B 2016, 93, 235162 .

(34) Rackers, J.; Wang, Z.; Lu, C.; Laury, M. L.; Lagardère, L.; Schnieders, M. J.; Piquemal, J.-P.; Ren, P.; Ponder, J. W. Tinker 8: Software Tools for Molecular Design. J. Chem. Theory Comput. 2018, 14, 5273-5289.

(35) Clancy, P. Application of Molecular Simulation Techniques to the Study of Factors Affecting the Thin-Film Morphology of Small-Molecule Organic Semiconductors. Chem. Mater. 2011, 23, 522-543.

(36) DuBay, K. H.; Hall, M. L.; Hughes, T. F.; Wu, C.; Reichman, D. R.; Friesner, R. A. Ac- 
curate Force Field Development for Modeling Conjugated Polymers. J. Chem. Theory Comput. 2012, 8, 4556-4569.

(37) Marcon, V.; Raos, G. Molecular Modeling of Crystalline Oligothiophenes: Testing and Development of Improved Force Fields. J. Phys. Chem. B 2004, 108, 18053-18064.

(38) Breiby, D. W.; Bunk, O.; Andreasen, J. W.; Lemke, H. T.; Nielsen, M. M. Simulating X-ray diffraction of textured films. J. Appl. Cryst. 2008, 41, 262-271.

(39) Resel, R.; Bainschab, M.; Pichler, A.; Dingemans, T.; Simbrunner, C.; Stangl, J.; Salzmann, I. Multiple scattering in grazing-incidence X-ray diffraction: Impact on latticeconstant determination in thin films. J Synchrotron Radiat. 2016, 23.

(40) Heffelfinger, C. J.; Burton, R. L. X-Ray determination of the crystallite orientation distributions of polyethylene terephthalate films. Journal of Polymer Science 1960, $47,289-306$.

(41) Because of the powder averaging around the $\mathbf{a}^{*}$ reciprocal lattice vectors each $(h k \ell)$ reflection [except the $(h 00) \mathrm{s}$ ] forms a ring in reciprocal space. 


\title{
Grapical TOC Entry, For Table of Contents Use Only
}

\author{
Modelling of Grazing-Incidence X-ray Diffraction \\ from Naphthyl End-capped Oligothiophenes in \\ Organic Field-Effect Transistors \\ Michael J Winokur, ${ }^{* \dagger, \ddagger}$ Mathias K. Huss-Hansen, ${ }^{\ddagger}$ Andreas E. Lauritzen,, $\boldsymbol{q}^{\ddagger}$ \\ Mika Torkkeli, ${ }^{\ddagger}$ Jakob Kjelstrup-Hansen ${ }^{\S}$ and Matti Knaapila ${ }^{\ddagger}$ \\ $\dagger$ Department of Physics, University of Wisconsin, Madison, Wisconsin 53706 \\ $\ddagger$ Department of Physics, Technical University of Denmark, 2800 Kgs. Lyngby, Denmark \\ \Department of Physics, University of Oxford, OX1 3PU Oxford, United Kingdom \\ $\S N a n o S Y D$, Mads Clausen Institute, University of Southern Denmark, 6400 Sønderborg, \\ Denmark
}

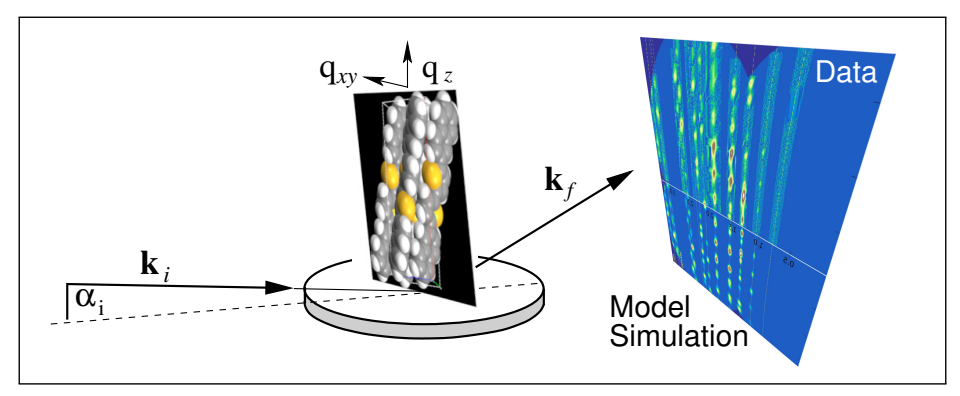

Depicted is a grazing incidence X-ray diffraction 2D image of scattering from a naphthylenecapped oligothiophene, 5,5'-bis(naphth-2-yl)-2,2'-bithiophene, thin-film field-effect transistor assembly in comparison with an analogous scattering pattern as obtained by molecular modelling in conjunction with structure factor refinement methods. 


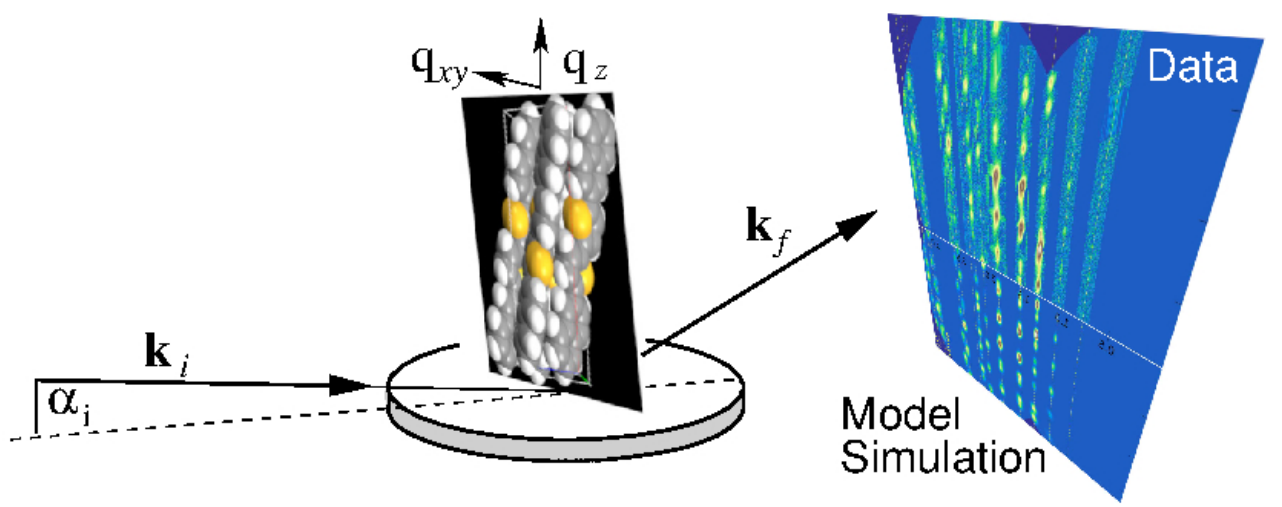

Depicted is a grazing incidence $X$-ray diffraction 2D image of scattering from a naphthylene-capped

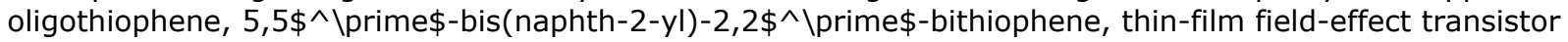
assembly in comparison with an analogous scattering pattern as obtained by molecular modelling in conjunction with structure factor refinement methods.

$269 \times 105 \mathrm{~mm}(80 \times 80 \mathrm{DPI})$ 